\title{
Regeneração e riqueza da formação arbustiva de Palmae em uma cronoseqüência pós-fogo na Restinga da Marambaia, Rio de Janeiro, RJ, Brasil
}

\author{
Luis Fernando Tavares de Menezes ${ }^{1,3}$ e Dorothy Sue Dunn de Araujo²
}

Recebido em 15/10/2003. Aceito em 08/04/2004

\begin{abstract}
RESUMO - (Regeneração e riqueza da formação arbustiva de Palmae em uma cronoseqüiência pós-fogo na Restinga da Marambaia, Rio de Janeiro, RJ, Brasil). A estrutura e a riqueza da formação arbustiva de Palmae foram analisadas em três sítios numa cronoseqüência de regeneração (3, 12 e 84 meses após a última queimada) na Restinga da Marambaia, registrando-se a presença de 29, 41 e 64 táxons, respectivamente. No sítio com maior tempo de regeneração, Allagoptera arenaria (Gomes) Kuntze representou 79\% da dominância relativa (DoR), seguida das nanofanerófitas Inga maritima Benth. e Manilkara subsericea (Mart.) Dubard. Na área queimada há 12 meses, A. arenaria representou 88\% da DoR, seguida de Inga maritima, Setaria setosa (Sw.) P. Beauv. e Paspalum arenarium Schrad. No sítio com três meses de regeneração, a DoR de A. arenaria foi de $82 \%$, acompanhada de Clitoria sp., Inga maritima e Portulaca mucronata Link. Nos três sítios estudados, a forma de vida mais importante foi geófita rizomatosa, devido à dominância de A. arenaria . Caméfita herbácea escaposa foi a forma de vida que apresentou maior número de espécies nos sítios com três e 12 meses de regeneração e no sítio queimado há 84 meses, as nanofanerófitas acompanharam as caméfitas herbáceas escaposas em número de espécies. Das 29 espécies registradas no sítio com três meses de regeneração, só Portulaca mucronata e Sebastiania corniculata (Vahl) Müll. Arg. originaram-se a partir de sementes, sendo que as demais rebrotaram ou se regeneraram. Sete dias após a queimada A. arenaria apresentou, em média, 8cm de sua parte vegetativa regenerada e com 180 dias apresentou as primeiras inflorescências.
\end{abstract}

Palavras-chave: sucessão secundária, rebrota, fogo, Allagoptera arenaria, restinga

\begin{abstract}
The structure and diversity of three areas of shrubby restinga vegetation were analyzed 3, 12 and 84 months after fire in the Marambaia Restinga, Rio de Janeiro State, Brazil). A total of 29, 41 and 64 taxa, respectively, were found. In the area sampled 84 months after fire, relative dominance of Allagoptera arenaria (Gomes) Kuntze was 79\%, followed by the nanophanerophytes Inga maritima Benth. and Manilkara subsericea (Mart.) Dubard. In the area sampled 12 months after fire, relative dominance of A. arenaria was 88\%, followed by Inga maritima, Setaria setosa (Sw.) P. Beauv and Paspalum arenarium Schrad. In the area sampled three months after fire, relative dominance of $A$. arenaria was $82 \%$; other important species were Clitoria sp., Inga maritima and Portulaca mucronata Link. Rhizome-geophytes dominated the life form spectra in the three areas due to A. arenaria dominance. The highest percentage of species in the areas sampled three and 12 months after fire belonged to the category scapose herbaceous chamaephytes. In the area sampled 84 months after fire, both nanophanerophytes and scapose herbaceous chamaephytes were dominant. Of the 29 species recorded in the area sampled three months after fire, only Portulaca mucronata and Sebastiania corniculata (Vahl) Müll. Arg. arose from seed; the others resprouted. Mean vegetative regrowth of $A$. arenaria seven days after fire was $8 \mathrm{~cm}$; after 180 days, this plant reproduced sexually.
\end{abstract}

Key words: secondary succession, sprouting, fire, Allagoptera arenaria, sandy coastal plain

\section{Introdução}

Desde os primeiros estudos sobre classificação de formações vegetais de restingas no litoral do Estado do Rio de Janeiro, a palmeira Allagoptera arenaria já era referida como sendo dominante em determinados trechos do cordão arenoso (Ule 1967; Dansereau 1947; Magnanini 1954). Posteriormente, esses trechos foram classificados por Araujo \& Henriques (1984) como “scrub” de Palmae (tratados aqui como formação arbustiva de Palmae - sensu Almeida \& Araujo 1997). Atualmente, essa formação é facilmente encontrada no cordão externo do sistema duplo de cordões arenosos das restingas do Rio de Janeiro (Massambaba, Jacarepaguá e Marambaia) e do Espírito Santo (Setiba, Ulé e Carais) (Pereira 1990; Araujo 1992). Estudos quantitativos realizados na formação arbustiva de Palmae confirmaram

\footnotetext{
1 Departamento de Botânica, Instituto de Biociências, Universidade Federal Rural do Rio de Janeiro, Antiga rodovia Rio-São Paulo, km 47, Seropédica, CEP 23851-970, Rio de Janeiro, RJ, Brasil

2 Departamento de Ecologia, Instituto de Biociências, Centro de Ciências da Saúde, Universidade Federal do Rio de Janeiro, C. Postal 68.020, Ilha do Fundão, CEP 21941-590, Rio de Janeiro, RJ, Brasil

3 Autor para correspondência: lftavares@br.inter.net
} 
A. arenaria como sendo a espécie de maior valor de importância em Saquarema (RJ), acompanhada de Diodea radula e Eugenia neonitida (Almeida \& Araujo 1997) e em Marambaia (RJ), acompanhada de Inga maritima e Manilkara subsericea (Menezes \& Araujo 1999), propiciando diferentes fisionomias no litoral do sudeste brasileiro.

Allagoptera arenaria é uma espécie de caule subterrâneo (Tomlinson 1960; Rizzini 1979; Leite 1990; Moraes 1996) que suporta a ação das queimadas na restinga (Araujo \& Peixoto 1977; Almeida \& Araujo 1997; Cirne \& Scarano 1996; Menezes \& Araujo 2000). Por essa razão, têm-se sugerido que a formação arbustiva de Palmae deva sua existência a queimadas periódicas, substituindo formações vegetais mais densas e fechadas, como a de pós-praia ou a de Myrtaceae, por exemplo (Araujo 1992; Lacerda et al. 1993). Entretanto, conhece-se pouco sobre as causas para o aspecto adensado que algumas populações de A. arenaria assumem em determinadas restingas, como na Marambaia.

Informações sobre processos sucessionais após distúrbios antrópicos em ecossistemas de restinga no Brasil ainda são restritas, sendo menores ainda aquelas referentes à influência do fogo sobre a vegetação. Estudos a esse respeito restringem-se aos de Araujo \& Peixoto (1977), que analisaram uma vegetação aberta herbáceo-arbustiva em Jacarepaguá (RJ) logo após a passagem do fogo; aos de Cirne \& Scarano (1996; 2001), que estudaram o rebrotamento de Andira legalis após uma queimada, na restinga de Jacarepiá - Saquarema (RJ) e aos de Cirne et al. (2003), que analisaram a diversidade de plantas e a associação interespecífica após duas queimadas num período de dez anos, também em Jacarepiá. Outros estudos, não tendo o fogo como principal agente modificador da vegetação, foram realizados no Rio de Janeiro por Sá (1996; 2002) e Gonçalves \& Sá (1998), que analisaram a dinâmica de regeneração da floresta de restinga após perturbação por tratores e por Araujo et al. (1997), que compararam a estrutura da vegetação e condições edáficas de uma clareira com a floresta de restinga contígua. Na Paraíba, Carvalho \& Oliveira-Filho (1993) e Miranda et al. (1997) avaliaram a recomposição da cobertura vegetal em dunas de rejeito de mineração. Recentemente, Carvalhaes \& Mantovani (1998) analisaram a composição florística de uma restinga em São Paulo utilizada até 35 anos atrás para o cultivo agrícola. Dentro da situação de devastação em que se encontram as formações vegetais de restinga no litoral brasileiro, é necessário conhecer-se os processos de regeneração após distúrbios causados pelo homem para a criação de parâmetros que auxiliem a recuperação de áreas descaracterizadas.

Considerando que o fogo é um dos principais fatores que pode alterar a estrutura, composição florística e recrutamento de plantas em uma comunidade vegetal (Forman \& Boerner 1981; Frost \& Robertson 1985; Frost et al. 1986; Woods 1989; Moreira 1996; Tester 1996) este trabalho teve como objetivo responder as seguintes questões: (i) Existem diferenças florísticas e estruturais em áreas da formação arbustiva de Palmae com diferentes períodos de regeneração após queimada? (ii) Quais as formas de vida mais importantes e freqüentes nessas áreas?

\section{Material e métodos}

Área de estudo - A Restinga da Marambaia, com aproximadamente $49,40 \mathrm{~km}^{2}$, localiza-se no Estado do Rio de Janeiro, compreendendo os municípios do Rio de Janeiro, Itaguaí e Mangaratiba, entre os paralelos $23^{\circ} 01^{\prime} \mathrm{S}$ e $23^{\circ} 06^{\prime} \mathrm{S}$ e meridianos $43^{\circ} 33^{\prime} \mathrm{W}$ e $44^{\circ} 01^{\prime} \mathrm{W}$. A formação arbustiva de Palmae nessa região compreende uma faixa com cerca de 703,62ha (M.H.B. Góes, dados não publicados) que se estende por quase toda a restinga, geralmente localizada adjacente à formação psamófila-reptante. A ocorrência do fogo na formação arbustiva de Palmae é comum nesta faixa, porém não a afeta de maneira homogênea, caracterizando um mosaico formado por trechos de diferentes tamanhos e idades após a última queimada. A ação do fogo é rápida, queimando a parte aérea da palmeira formada por folhas verdes e a serapilheira composta quase que exclusivamente por suas folhas senescentes depositadas sob a copa da planta.

O trecho da formação arbustiva de Palmae estudado localiza-se na extremidade leste da Restinga da Marambaia, entre a Estação Meteorológica e a Linha II, aproximadamente $7 \mathrm{~km}$ a oeste da ponte que liga a restinga ao continente. Este trecho, de fisionomia homogênea, estende-se numa faixa estreita com cerca de $5 \mathrm{~km}$ compr. e é interceptado ao meio por uma estrada. É delimitado ao sul pela comunidade psamófilareptante, próximo ao mar e ao norte, pela vegetação densa e contínua, com cerca de $6 \mathrm{~m}$ alt., que recobre as dunas (Menezes et al. 1998). Este trecho foi estudado sete anos após a queimada de 1989 quanto ao aspecto estrutural (Menezes \& Araujo 1999) e quanto à disponibilidade de biomassa aérea de A. arenaria (Menezes \& Araujo 2000). 
Clima - A área da Restinga da Marambaia, segundo a classificação de Köeppen (1948), enquadra-se no macroclima Aw (Clima Tropical Chuvoso), com temperaturas do ar típicas das áreas litorâneas tropicais. A temperatura média anual é de $23,7^{\circ} \mathrm{C}$, sendo o mês de fevereiro o mais quente do ano (média de $26,8^{\circ} \mathrm{C}$ ) e agosto o mais frio (média de $20,9^{\circ} \mathrm{C}$ ). $\mathrm{O}$ climatograma obtido para a Restinga da Marambaia mostra que não ocorrem meses secos durante o ano, caracterizando o clima como super-úmido (Fig. 1). A precipitação média anual é de $1.239,7 \mathrm{~mm}$, onde $37 \%$ deste valor ocorre no verão, distribuídos em torno de 40 dias chuvosos. Os meses de inverno são os mais secos, quando ocorre apenas $15 \%$ da precipitação média anual, distribuída ao redor de 21 dias. Em julho e agosto a precipitação média mensal situa-se entre 40 e 55mm, já tendo por diversos anos ocorridos valores abaixo de $30 \mathrm{~mm}$, ocasionando acentuada seca na região (C.L. Mattos, dados não publicados).

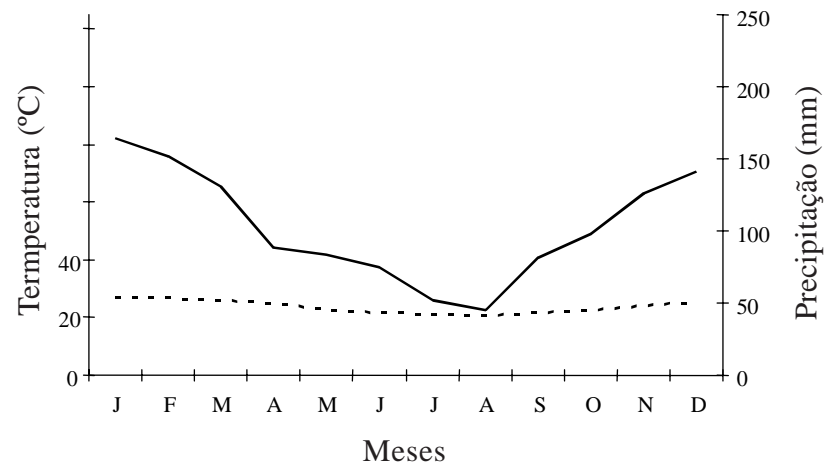

Figura 1. Diagrama Ombrotérmico de Gaussen-Bagnouls para a Restinga da Marambaia, RJ. Período: 1986-1998. Fonte: C.L. Mattos, dados não publicados. - - - Temperatura; - Precipitação.

Amostragem - Duas novas ocorrências de fogo foram registradas em partes do trecho analisado por Menezes \& Araujo (1999), sendo as áreas atingidas classificadas quanto ao tempo de regeneração após a última queimada: sítio 0,25 - PF (o número faz alusão ao tempo de regeneração da vegetação após a última queimada, estimado em anos, designado pós-fogo - PF) localizado nas coordenadas $23^{\circ} 03^{\prime} 0,6^{\prime \prime}$ 'S e 4338'76,3' W e distante cerca de $300 \mathrm{~m}$ do mar. Possui $1 \mathrm{~km}$ de extensão e $150 \mathrm{~m}$ de largura, tendo sido queimado pela última vez em 21/setembro/1997 e analisado estruturalmente três meses após; sítio 1-PF, distante do primeiro $300 \mathrm{~m}$, a leste (2302'98,5”'S; 4338'50,2”W) com cerca de 500m de extensão e 50-60m de largura. Foi queimado novamente em maio/1996 e estudado um ano após.
Considerou-se como sítio de controle o trecho ausente de fogo durante sete anos (7-PF), estudado por Menezes \& Araujo (1999).

Adotou-se a denominação “ilha de vegetação” para os adensamentos de Allagoptera arenaria, independentemente da presença ou ausência de indivíduos com ramificações subterrâneas. A confirmação de indivíduos ramificados formando unidades modulares com conexões entre si foi feita através de escavações em "ilhas de vegetação" formada pela palmeira nos sítios 1-PF e 7-PF. Neste caso, o termo crescimento clonal foi adotado (Cook 1983).

A análise estrutural da vegetação foi realizada pelo método das parcelas (Müeller-Dumbois \& Ellenberg 1974) ao longo de 8, 10 e 13 linhas perpendiculares ao mar, estabelecidas nos sítios 0,25 PF, 1-PF e 7-PF, respectivamente, distantes uma da outra em $15 \mathrm{~m}$ nos sítios 0,25-PF e 1-PF. No sítio 7-PF a distância entre as linhas foi variável. Ao longo das linhas foram distribuídas, em lados alternos, parcelas de $1 \mathrm{~m}^{2}$ com intervalos de $1 \mathrm{~m}$ entre elas, perfazendo o total de 200, 277 e $403 \mathrm{~m}^{2}$, nos sítios 0,25-PF, 1-PF e 7-PF, respectivamente. A diferença entre os tamanhos dos sítios amostrais foi em detrimento dos diferentes tamanhos das áreas atingidas pelo fogo. Em cada parcela foi estimada visualmente a cobertura das espécies (área ocupada pela projeção da parte aérea da planta, estimada como uma percentagem e expressa em $\mathrm{m}^{2}$ - Brower \& Zar 1984). Os valores relativos de dominância (cobertura) e freqüência foram utilizados para determinar o valor de importância (VI). A densidade não foi determinada devido à dificuldade de individualização das plantas, principalmente Allagoptera arenaria. Müeller-Dumbois \& Ellenberg (1974) consideraram as estimativas de cobertura e freqüência suficientes para a análise descritiva de formações herbáceas ou baixo-arbustivas, sendo desnecessário o esforço demandado para a determinação da densidade. Este procedimento foi adotado por Almeida \& Araujo (1996), Pereira et al. (1992) e Menezes \& Araujo (1999) em estudos estruturais em restingas onde a vegetação apresenta características similares às do presente estudo.

No sítio 0,25-PF, devido à facilidade de observação e acompanhamento, foram registradas as espécies que rebrotavam e aquelas que se estabeleciam por sementes, o que era confirmado por meio de escavações, quando necessário. Espécies carbonizadas e que não apresentavam rebrotas tiveram partes dos ramos cortados e seus sistemas subterrâneos investigados para a confirmação da morte dos mesmos. 
Para estimar o crescimento de Allagoptera arenaria após o fogo foi mensurado no sítio $0,25-\mathrm{PF}$ a distância entre a superfície do solo e a extremidade do ápice da maior folha esticada ao máximo de 40 indivíduos escolhidos ao acaso, no período de 7, 41, 69 e 180 dias após a queimada de 21/setembro/1997. Com 180 dias após a queimada verificou-se o número total de folhas vivas presentes em cada um dos 40 indivíduos.

As variações fisionômicas entre os sítios com diferentes tempos de regeneração após o fogo foram observadas por meio da classificação das formas de vida das espécies vegetais presentes em cada um dos sítios analisados, segundo critérios apresentados por Müeller-Dombois \& Ellenberg (1974).

\section{Resultados e discussão}

Crescimento e rebrota das espécies - Todos os indivíduos de $A$. arenaria $(\mathrm{N}=194)$ amostrados no sítio 0,25-PF apresentaram resistência ao fogo. Sete dias após a queimada, folhas jovens carbonizadas apresentaram em média $8 \mathrm{~cm}$ de tecido foliar novo, emergido da base do pecíolo de todas as plantas afetadas pelo fogo (Tab. 1). Essa resistência envolve a preservação da gema principal que se localiza abaixo do solo. Ao germinar, A. arenaria aprofunda seu caule por geotropismo positivo alguns centímetros no solo, para em seguida reverter seu crescimento em direção à superfície (L.F.T. Menezes, dados não publicados). Provavelmente, o fogo não chega a proporcionar altas temperaturas no solo, ficando as gemas protegidas, danificando apenas a parte aérea da planta. Mesmo na savana, onde a temperatura do ar no ponto máximo das chamas pode atingir valores da ordem de $800^{\circ} \mathrm{C}$, em profundidades de dois a cinco centímetros abaixo da superfície do solo, a temperatura eleva-se apenas alguns graus devido ao poder isolante do solo, resguardando os órgãos subterrâneos das plantas (Coutinho 1982; Ramos \& Rosa 1992). Entretanto, no

Tabela 1. Crescimento médio das folhas de Allagoptera arenaria, em diferentes períodos de regeneração após queimada na formação arbustiva de Palmae, na Restinga da Marambaia, Rio de Janeiro, RJ.

\begin{tabular}{ccc}
\hline $\begin{array}{c}\text { N. de dias após a } \\
\text { queimada }\end{array}$ & $\begin{array}{c}\text { Média do crescimento } \\
(\mathrm{cm}) \mathrm{N}=40\end{array}$ & Desvio padrão \\
\hline 7 & 8 & 0,91 \\
41 & 43 & 5,12 \\
69 & 68 & 8,86 \\
180 & 81 & 8,64 \\
\hline
\end{tabular}

sítio 0,25-PF quatro arbustos encontravam-se mortos, provavelmente por efeitos da ação do fogo, não sendo possível o reconhecimento da espécie.

As escavações realizadas em "ilhas de vegetação" de A. arenaria revelaram indivíduos com até quatro ramificações laterais, ligadas de maneira muito próxima a um caule mais desenvolvido. É possível que a destruição da parte aérea da planta pelo fogo induza atividades meristemáticas em tecidos de reservas do eixo principal do sistema subterrâneo, originando indivíduos ramificados, contribuindo para o aspecto adensado da formação. Cirne \& Scarano (2001) observaram que todos os indivíduos de Andira legalis (Vell.) Toledo atingidos pelo fogo sobreviveram e aumentaram o número de rametes após uma queimada na restinga de Jacarepiá (RJ). Esses autores sugerem que alterações fisiológicas controladas internamente pela planta estimulam meristemas de estruturas subterrâneas a produzirem novos brotos.

$\mathrm{O}$ crescimento novo das folhas de A. arenaria foi maior até 69 dias após a queimada (Tab. 1). Entre 69 e 180 dias apresentou pouco crescimento. Neste intervalo, os indivíduos ( $\mathrm{N}=40)$ continham, em média, quatro folhas e desses, dois encontravam-se floridos, mostrando que nesse período a planta recupera a capacidade reprodutiva após a queimada. De fato, em espécies de crescimento clonal são comuns a rápida rebrota e a alta taxa de crescimento, permitindo a rápida recolonização de ambientes sujeitos ao fogo (Kauffman 1991; De Rouw 1993; Stuefer 1994; Nascimento 1996; Pornon et al. 1997), conferindo-lhes vantagens adaptativas à ocupação do hábitat após as queimadas (Whelan 1995; Goto et al. 1996).

Embora o fogo seja incomum nas restingas, a regeneração da vegetação observada aqui é uma estratégia comum em diversos ecossistemas compostos por plantas adaptadas à ação do fogo (Bond \& van Wilgen 1996; Whelan 1995). No sítio 0,25-PF, com exceção de Portulaca mucronata e Sebastiania corniculata que se estabeleceram a partir de sementes, todas as outras espécies registradas $(\mathrm{N}=27)$, originaram-se a partir de rebrotas de estruturas subterrâneas. Fato semelhante foi observado por Araujo \& Peixoto (1977) na restinga de Jacarepaguá (RJ), onde o total de 19 espécies surgidas 30 dias após a queimada era originado de rebrotas de órgãos vegetativos subterrâneos. Em sistemas florestais, a estratégia de rebrota de indivíduos pré-estabelecidos na área após a ocorrência de fogo, parece ser relativamente comum (Castellani \& Stubblebine 1993; Tabarelli \& Mantovani 1999). 
Composição florística e estrutura - As espécies ocorrentes nos três sítios estudados estão indicadas na Tabela 2. O número total de espécies em cada sítio aumentou em relação ao do tempo de regeneração decorrido após a última queimada (29, 41 e 64 nos sítios 0,25-PF, 1-PF e 7-PF, respectivamente). Araujo \& Peixoto (1977) também observaram que para um período de 3, 11, 24 e 30 meses após uma queimada na restinga de Jacarepaguá (RJ), o número de espécies aumentou de 19 para 38, 46 e 52, respectivamente. Esses autores ressaltam a importância de A. arenaria durante o processo sucessional, protegendo o solo da intensa radiação solar, facilitando o estabelecimento de outras espécies.

Na Marambaia, o aumento da riqueza específica, de acordo com a cronoseqüência após a última queimada, pode ser decorrente das modificações no ambiente proporcionadas por A. arenaria. A matéria orgânica provida da senescência contínua de suas folhas aumenta as condições nutricionais do solo ao longo do tempo (Hay et al. 1982; Menezes \& Araujo 2000) que, por sua vez, acarretam mudanças nas características químicas do mesmo, fundamentais nos processos de sucessão ecológica (Etherington 1967; Andrade 1971; Denslow 1980; Hay \& Lacerda 1980). Estas condições microclimáticas desenvolvidas por A. arenaria ao longo do tempo, além do sombreamento causado por sua copa e, conseqüentemente, diminuição da temperatura do solo abaixo dela podem aumentar as condições para o estabelecimento de novas espécies (Araujo \& Peixoto 1977; Huston \& Smith 1987; Menezes \& Araujo 2000). De fato, o reduzido teor de nutrientes no solo e a dessecação de sementes e plântulas são as causas primárias que limitam o estabelecimento de espécies em áreas desnudas nas restingas (Maun 1994). Estas condições parecem enquadrar-se no processo clássico de facilitação indireta (sensu Callaway 1995), quando a presença de uma dada espécie aumenta a sobrevivência, crescimento ou valor adaptativo de outra. A possibilidade de novas associações entre A. arenaria e outras espécies traz um novo eixo explicativo que pode ser explorado em estudos de interações positivas (Alpert \& Mooney 1996; Pugnaire et al. 1996; Zaluar \& Scarano 2001; Scarano 2002; Scarano et al. 2004).

Todas as espécies dos sítios 0,25-PF e 1-PF ocorreram no sítio 7-PF (Tab. 2). Entretanto, cinco espécies do sítio 0,25-PF não ocorreram no sítio 1-PF (Smilax rufescens, Remirea marítima, Borreria verticillata e as nanofanerófitas Eugenia rotundifolia

Tabela 2. Espécies ocorrentes nos três sítios da formação arbustiva de Palmae da restinga da Marambaia, Rio de Janeiro, RJ, analisados após a ocorrência de fogo (0,25-PF, com 3 meses de regeneração; 1-PF, com 1 ano de regeneração e 7- PF, com 7 anos de regeneração). As formas de vidas (FV) estão indicadas por: Geófita rizomatosa (Gz), Nanofanerófita (Na), Caméfita herbácea escaposa (Che), Trepadeira geofítica (Tg), Caméfita suculenta (Cs), Geófita radicigema (Gr), Hemicriptófita escaposa rosetada (Her), Caméfita herbácea reptante (Chr), Fanerófitas suculentas (Fs), Caméfita herbácea pulvinada (Chp), Liana fanerófita (Lf) e Epífita (Ep).

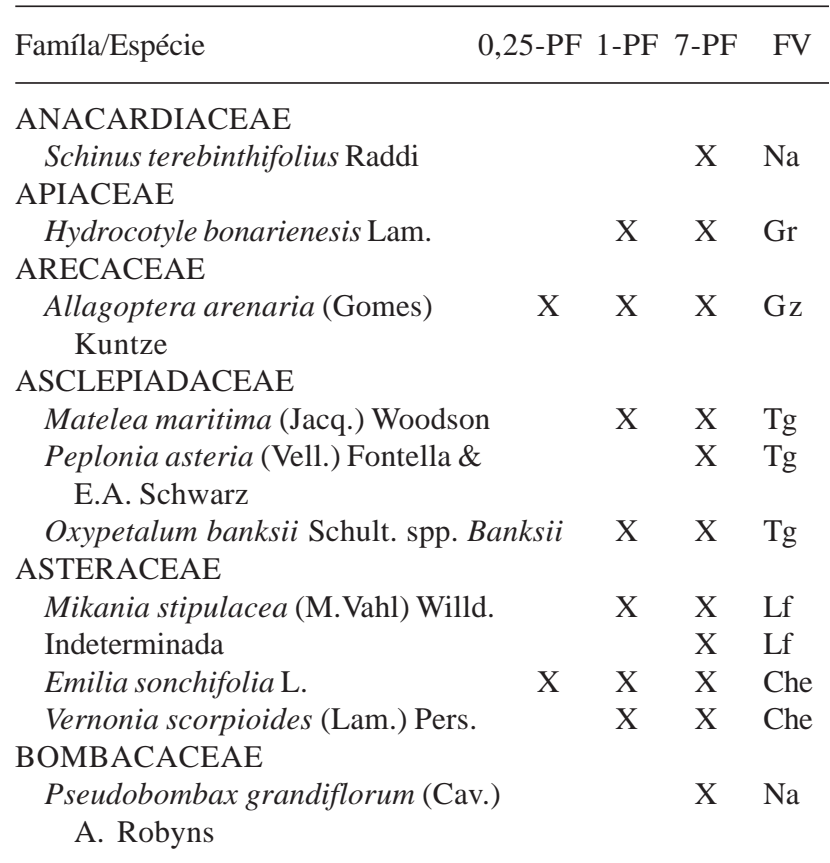

\begin{tabular}{|c|c|c|c|c|}
\hline Famíla/Espécie & 5-PF & $1-\mathrm{PF}$ & 7-PF & FV \\
\hline \multicolumn{5}{|l|}{ BORAGINACEAE } \\
\hline Tournefortia villosa Salzm. & & $\mathrm{X}$ & $\mathrm{X}$ & $\operatorname{Tg}$ \\
\hline \multicolumn{5}{|l|}{ BROMELIACEAE } \\
\hline Neoregelia cruenta(Graham) L.B. Sm. & $\mathrm{X}$ & $\mathrm{X}$ & $\mathrm{X}$ & Her \\
\hline $\begin{array}{l}\text { Quesnelia quesneliana } \\
\text { (Brongn.) L.B. Sm. }\end{array}$ & & & $\mathrm{X}$ & Her \\
\hline Bromelia antiacantha Bertol. & & & $\mathrm{X}$ & Her \\
\hline \multicolumn{5}{|l|}{ CACTACEAE } \\
\hline $\begin{array}{l}\text { Pilosocereus arrabidae (Lem.) Byles } \\
\text { \& G.D. Rowley }\end{array}$ & & $\mathrm{X}$ & $\mathrm{X}$ & Fs \\
\hline Cereus fernambucensis Lem. & $\mathrm{X}$ & $\mathrm{X}$ & $\mathrm{X}$ & Cs \\
\hline \multicolumn{5}{|l|}{ CALYCERACEAE } \\
\hline Acicarpha spatulata R. Br. & & $\mathrm{X}$ & & Chr \\
\hline \multicolumn{5}{|l|}{ CONVOLVULACEAE } \\
\hline Ipomoea imperati (Vahl.) Griseb. & & & $\mathrm{X}$ & $\mathrm{Gr}$ \\
\hline Ipomoea pes-capre (L.) Sweet. & & $\mathrm{X}$ & $\mathrm{X}$ & Chr \\
\hline \multicolumn{5}{|l|}{ CYPERACEAE } \\
\hline $\begin{array}{l}\text { Jacquemontia holosericea } \\
\text { (Weinm.) O’Donell }\end{array}$ & & & $\mathrm{X}$ & $\operatorname{Tg}$ \\
\hline Remirea maritima Aubl. & $\mathrm{X}$ & & $\mathrm{X}$ & Che \\
\hline
\end{tabular}


Tabela 2 (continuação)

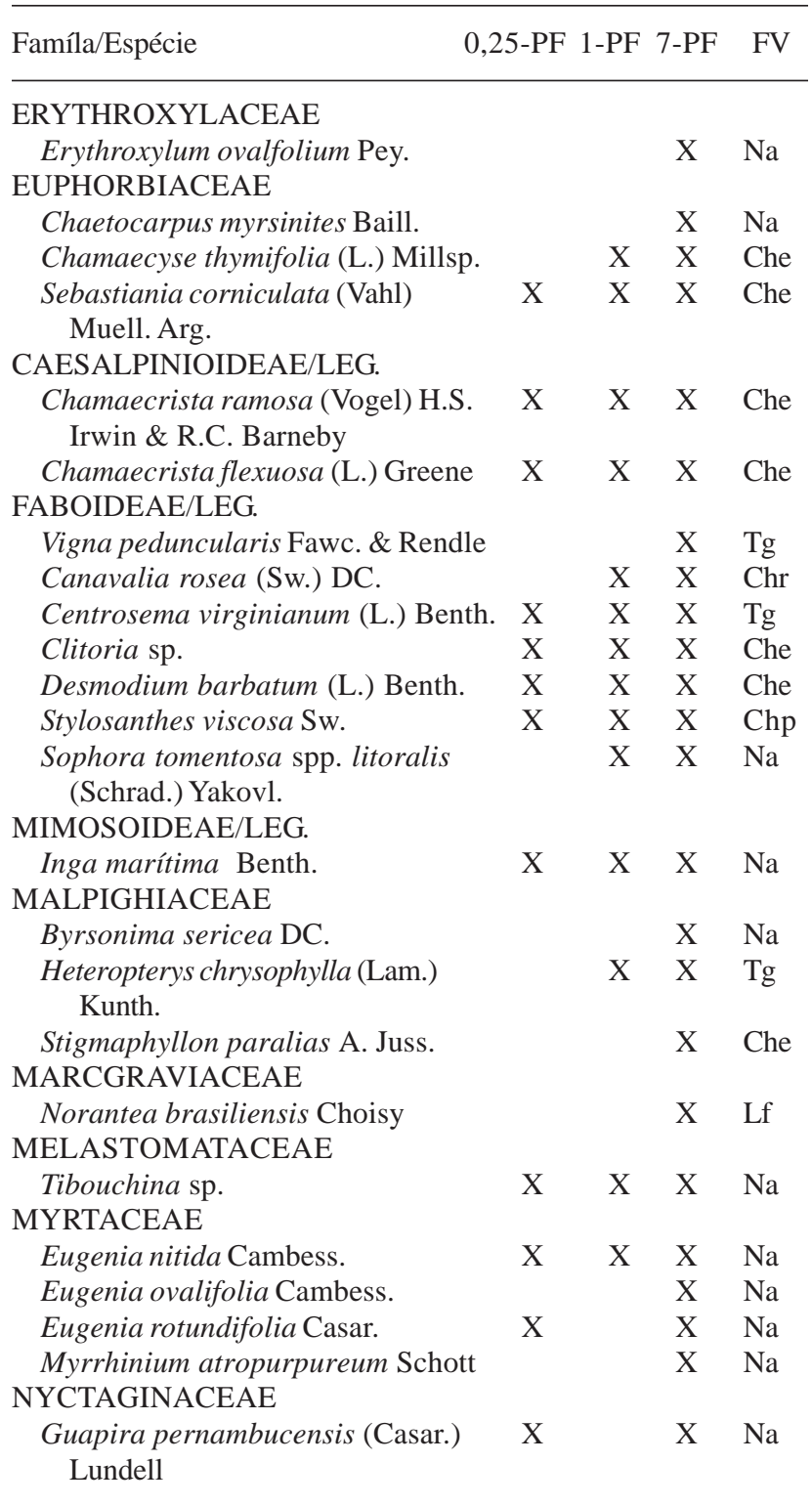

e Guapira pernambucensis). A exclusividade de Acicarpha spathulata no sítio 1-PF foi devido à localização do próprio sítio, que englobava trechos de transição entre as formações psamófila-reptante e arbustiva de Palmae, locais onde eventualmente esta espécie ocorre.

Nos três sítios analisados, $A$. arenaria foi a espécie que apresentou maior valor de cobertura total e freqüência relativa (Tab. 3). Conseqüentemente, maiores valores desses parâmetros conferem a esta espécie maior valor de importância, fato este já observado para outras formações de Palmae estudadas (Almeida \& Araujo 1997). Para a Marambaia, a dominância relativa de $A$. arenaria oscilou de 82 a 88 e $79 \%$ nos sítios

\begin{tabular}{|c|c|c|c|c|}
\hline Famíla/Espécie & 5-PF & $1-\mathrm{PF}$ & 7-PF & FV \\
\hline \multicolumn{5}{|l|}{ OCHNACEAE } \\
\hline Ouratea cuspidata (A. St.-Hil.) Engl. & & & $\mathrm{X}$ & $\mathrm{Na}$ \\
\hline \multicolumn{5}{|l|}{ ORCHIDACEAE } \\
\hline $\begin{array}{l}\text { Cyrtopodium polyphyllum (Vell.) } \\
\text { Pabst ex F. Barros }\end{array}$ & $\mathrm{X}$ & $\mathrm{X}$ & $\mathrm{X}$ & $\mathrm{Gr}$ \\
\hline Epidendrum denticulatum Barb. Rodr. & $\mathrm{X}$ & $\mathrm{X}$ & $\mathrm{X}$ & Ep \\
\hline $\begin{array}{l}\text { Vanilla chamissonis var. brevifolia } \\
\text { Cogn. }\end{array}$ & & $\mathrm{X}$ & $\mathrm{X}$ & $\operatorname{Tg}$ \\
\hline Catasetum discolor (Lindl.) Lindl. & & & $\mathrm{X}$ & $\mathrm{Gr}$ \\
\hline \multicolumn{5}{|l|}{ POACEAE } \\
\hline $\begin{array}{l}\text { Panicum racemosum (P. Beauv.) } \\
\text { Spreng. }\end{array}$ & $\mathrm{X}$ & $\mathrm{X}$ & $\mathrm{X}$ & $\mathrm{Gz}$ \\
\hline Setaria setosa (Swartz) P. Beauv. & & $\mathrm{X}$ & $\mathrm{X}$ & $\mathrm{Gz}$ \\
\hline $\begin{array}{l}\text { Stenotaphrum secundatum } \\
\text { (Walter) Kuntze }\end{array}$ & & $\mathrm{X}$ & $\mathrm{X}$ & Chr \\
\hline Paspalum arenarium Schrad. & $\mathrm{X}$ & $\mathrm{X}$ & $\mathrm{X}$ & $\mathrm{Gz}$ \\
\hline Paspalum vaginatum Sw. & & $\mathrm{X}$ & $\mathrm{X}$ & $\mathrm{Gz}$ \\
\hline Sporobolus virginicus (L.) Kunth & & & $\mathrm{X}$ & $\mathrm{Gz}$ \\
\hline \multicolumn{5}{|l|}{ PORTULACACEAE } \\
\hline Portulaca mucronata Link & $\mathrm{X}$ & $\mathrm{X}$ & $\mathrm{X}$ & Che \\
\hline \multicolumn{5}{|l|}{ RUBIACEAE } \\
\hline $\begin{array}{l}\text { Borreria brachystemonoides Cham. } \\
\text { \& Schltdl. }\end{array}$ & $\mathrm{X}$ & $\mathrm{X}$ & $\mathrm{X}$ & Che \\
\hline Borreria verticillata (L.) Meyer & $\mathrm{X}$ & & $\mathrm{X}$ & Che \\
\hline $\begin{array}{l}\text { Diodea radula (Willd. \& Hoffmanns.) } \\
\text { Cham. \& Schltdl. }\end{array}$ & $\mathrm{X}$ & $\mathrm{X}$ & $\mathrm{X}$ & Che \\
\hline Tocoyena bullata Mart. & $\mathrm{X}$ & $\mathrm{X}$ & $\mathrm{X}$ & $\mathrm{Na}$ \\
\hline Diodea conferta (Schott) DC. & $\mathrm{X}$ & $\mathrm{X}$ & $\mathrm{X}$ & Che \\
\hline \multicolumn{5}{|l|}{ SAPINDACEAE } \\
\hline Paullinea coriacea Casar. & & & $\mathrm{X}$ & $\operatorname{Tg}$ \\
\hline \multicolumn{5}{|l|}{ SAPOTACEAE } \\
\hline $\begin{array}{l}\text { Manilkara subsericea (Mart.) } \\
\text { Dubard }\end{array}$ & $\mathrm{X}$ & $\mathrm{X}$ & $\mathrm{X}$ & $\mathrm{Na}$ \\
\hline \multicolumn{5}{|l|}{ SMILACEAE } \\
\hline Smilax rufescens Griseb. & $\mathrm{X}$ & & $\mathrm{X}$ & $\operatorname{Tg}$ \\
\hline
\end{tabular}

0,25-PF, 1-PF e 7-PF, respectivamente, após a última queimada. Entretanto, é provável que o valor de cobertura da espécie aumente no sítio 0,25-PF, pois em 90 dias de regeneração as folhas das plantas ainda não tinham atingido o crescimento máximo. Em Jacarepaguá, esta espécie correspondeu a 16\% da cobertura vegetal, três meses após uma queimada, sendo elevada para 36\% após 30 meses (Araujo \& Peixoto 1977).

Setaria setosa e Inga marítima também foram espécies comuns aos três sítios e estiveram entre as 10 espécies de maior valor de importância em cada um deles. I. marítima e Manilkara subsericea (nanofanerófitas) tiveram comportamento similar ao de $A$. arenaria, ou seja, aumentaram a cobertura de 
Tabela 3. Parâmetros fitossociológicos das dez espécies mais importantes para os três sítios estudados da formação arbustiva de Palmae, na Restinga da Marambaia, Rio de Janeiro, RJ. NPe - Número de parcelas onde foi encontrada a espécie; CT - Cobertura total da espécie (m²/ha); FR - Freqüência relativa (\%); DoR - Dominância relativa (\%); VI - Valor de importância (\%). Os sítios 0,25-PF, 1-PF e 7-PF, indicam 3 meses, 1 e 7 anos de regeneração após fogo, respectivamente. Dados do sítio 7-PF, segundo Menezes \& Araujo (1999).

\begin{tabular}{|c|c|c|c|c|c|}
\hline \multirow[t]{2}{*}{ Espécies ocorrentes } & \multicolumn{5}{|c|}{ Parâmetros fitossociológicos } \\
\hline & Npe & $\mathrm{CT}$ & $\mathrm{FR}^{*}$ & DoR* & $\mathrm{VI}^{* *}$ \\
\hline \multicolumn{6}{|l|}{ Espécies do sítio 0,25-PF } \\
\hline Allagoptera arenaria & 194 & 4738 & 39,59 & 82,01 & 121,61 \\
\hline Clitoria sp. & 74 & 482 & 15,10 & 8,34 & 23,45 \\
\hline Portulaca mucronata & 52 & 62 & 10,61 & 1,07 & 11,69 \\
\hline Borreria brachystemonoides & 30 & 38 & 6,12 & 0,66 & 6,78 \\
\hline Inga maritima & 21 & 92 & 4,29 & 1,59 & 5,88 \\
\hline Chamaecrista flexuosa & 18 & 34 & 3,67 & 0,59 & 4,26 \\
\hline Setaria setosa & 12 & 62 & 2,45 & 1,07 & 3,52 \\
\hline Eugenia neonítida & 9 & 56 & 1,84 & 0,97 & 2,81 \\
\hline Borreria verticillata & 12 & 15 & 2,45 & 0,26 & 2,71 \\
\hline Paspalum arenarium & 11 & 23 & 2,24 & 0,40 & 2,64 \\
\hline \multicolumn{6}{|l|}{ Espécies do sítio 1-PF } \\
\hline Allagoptera arenaria & 249 & 10393 & 30,23 & 88,07 & 118,30 \\
\hline Paspalum arenarium & 72 & 156 & 8,74 & 1,32 & 10,06 \\
\hline Panicum racemosum & 56 & 33 & 6,80 & 0,28 & 7,08 \\
\hline Setaria setosa & 43 & 211 & 5,22 & 1,79 & 7,01 \\
\hline Desmodium barbatum & 48 & 74 & 5,83 & 0,63 & 6,45 \\
\hline Chamaecyce thymifolia & 47 & 69 & 5,71 & 0,58 & 6,29 \\
\hline Borreria brachystemonoides & 31 & 100 & 3,76 & 0,85 & 4,61 \\
\hline Centrosema virginianum & 33 & 61 & 4,01 & 0,52 & 4,52 \\
\hline Inga marítima & 17 & 285 & 2,06 & 2,42 & 4,48 \\
\hline Hydrocotyle bonariensis & 22 & 34 & 2,67 & 0,29 & 2,96 \\
\hline \multicolumn{6}{|l|}{ Espécies do sítio 7-PF } \\
\hline Allagoptera arenaria & 364 & 19691 & 32,10 & 78,89 & 110,99 \\
\hline Inga marítima & 65 & 1005 & 5,73 & 4,03 & 9,76 \\
\hline Manilkara subsericea & 23 & 591 & 2,03 & 2,37 & 4,40 \\
\hline Stylosanthes viscosa & 43 & 138 & 3,79 & 0,55 & 4,34 \\
\hline Setaria setosa & 44 & 74 & 3,88 & 0,30 & 4,18 \\
\hline Cereus fernambucensis & 37 & 205 & 3,26 & 0,82 & 4,08 \\
\hline Chamaecrista flexuosa & 39 & 95 & 3,44 & 0,38 & 3,82 \\
\hline Desmodium barbatum & 37 & 85 & 3,26 & 0,34 & 3,60 \\
\hline Panicum racemosum & 35 & 69 & 3,09 & 0,28 & 3,36 \\
\hline Vernonia scorpioides & 31 & 103 & 2,73 & 0,41 & 3,15 \\
\hline
\end{tabular}

$* \sum$ de todas as espécies por área $=100 \% ; * \sum$ de todas as espécies por área $=200 \%$.

acordo com a cronoseqüência após a queimada (Tab. 4). Em trechos de florestas, muitas espécies com valores de importância altos após o fogo são aquelas que apresentam vantagens significativas associadas à regeneração por rebrotas (Castellani \& Stubblebine 1993). De fato, indivíduos que rebrotam não têm que alocar recursos à formação de raízes, o que lhes conferem vantagens competitivas em relação aos que colonizam a área através de sementes.

Portulaca mucronata obteve posição de destaque quanto à freqüência relativa no sítio 0,25-PF $(10,61)$. Nos sítios 1-PF e 7-PF a freqüência relativa para esta espécie foi de 2,67 e 0,26, respectivamente. Essa diminuição mostra que tal espécie é menos freqüente nos sítios com maior tempo de regeneração, caracterizando um caso típico da perda do hábitat para outra espécie de maior êxito (Huston \& Smith 1987; Leigh 1992; Costa \& Silva 1993).

Formas de vida - Nos três sítios estudados a forma de vida mais importante foi geófita rizomatosa, devido à dominância de A. arenaria em todos eles (Tab. 5). Com exceção desta forma de vida, caméfita herbácea escaposa foi a mais importante nos sítios 0,25-PF e 1-PF e também aquela que apresentou maior número de espécies. No sítio 7-PF, tanto nanofanerófitas quanto caméfitas herbáceas escaposas tiveram papel importante na estrutura. Caméfitas herbáceas escaposas são plantas herbáceas que não crescem 
acima de 1 metro do solo, ou cujo sistema aéreo constitui um único eixo ortotrópico que morre periodicamente e retrocede a um sistema caulinar que permanece verde, pelo menos a 0,25m acima do solo (Müeller-Dumbois \& Ellenberg 1974). Espécies que possuem tais características, aparentemente, apresentam vantagens adaptativas em explorar ambientes sujeitos ao fogo na Restinga da Marambaia. Vale ressaltar que Portulaca mucronata e Sebastiania corniculata foram as únicas espécies originadas a partir de sementes no sítio 0,25-PF e que também apresentam esta forma de vida.

Os resultados deste trabalho mostram que A. arenaria e algumas espécies nanofanerófitas resistem à intensidade do fogo que ocorre na formação arbustiva de Palmae da Restinga da Marambaia. A posição sociológica de $A$. arenaria não é alterada devido as suas características regenerativas, porém de
Tabela 4. Espécies de maior cobertura total e comuns aos três sítios da formação arbustiva de Palmae, com diferentes períodos de regeneração após a última queimada, na Restinga da Marambaia, Rio de Janeiro, RJ. Os sítios 0,25-PF, 1-PF e 7-PF, indicam 3 meses, 1 e 7 anos de regeneração após fogo, respectivamente. Dados do sítio 7-PF, segundo Menezes \& Araujo (1999).

\begin{tabular}{lrrr}
\hline \multirow{2}{*}{ Espécies } & \multicolumn{2}{c}{ Cobertura } & \multicolumn{2}{c}{ Total } & \multirow{2}{*}{$\left(\mathrm{m}^{2} / \mathrm{ha}\right)$} \\
& \multicolumn{2}{c}{ Sítios em regeneração } \\
\cline { 2 - 4 } & $0,25-\mathrm{PF}$ & $1-\mathrm{PF}$ & $7-\mathrm{PF}$ \\
\hline Allagoptera arenaria & 4.738 & 10.393 & 19.691 \\
Inga marítima & 92 & 285 & 1.005 \\
Manilkara subsericea & 8 & 10 & 591 \\
Clitoria sp. & 482 & 41 & 89 \\
Setaria setosa & 62 & 211 & 74 \\
Paspalum arenarium & 23 & 156 & 69 \\
Borreria brachystemonoides & 38 & 100 & 40 \\
Portulaca mucronata & 62 & 27 & 5 \\
\hline
\end{tabular}

Tabela 5. Número de espécies e valor de importância, em cada tipologia de forma de vida, em diferentes sítios de regeneração após queima na formação arbustiva de Palmae da Restinga da Marambaia, Rio de Janeiro, RJ. (0,25-PF, sítio em regeneração há 3 meses; 1-PF, sítio em regeneração há 1 ano e 7-PF, sítio em regeneração há 7 anos).

\begin{tabular}{|c|c|c|c|c|c|c|}
\hline \multirow{3}{*}{ Formas de vida } & \multicolumn{6}{|c|}{ Sítios em regeneração } \\
\hline & \multicolumn{2}{|c|}{$0,25-\mathrm{PF}$} & \multicolumn{2}{|c|}{$1-\mathrm{PF}$} & \multicolumn{2}{|c|}{ 7-PF } \\
\hline & N. de espécies & $\begin{array}{l}\text { VI total } \\
(\%)^{*}\end{array}$ & N. de espécies & $\begin{array}{l}\text { VI total } \\
(\%)^{*}\end{array}$ & N. de espécies & $\begin{array}{l}\text { VI total } \\
(\%)^{*}\end{array}$ \\
\hline Geófita rizomatosa & 4 & 130,50 & 6 & 144,32 & 6 & 122,77 \\
\hline Nanofanerófita & 8 & 11,13 & 6 & 5,33 & 16 & 23,95 \\
\hline Caméfita herbácea escaposa & 10 & 52,59 & 13 & 34,32 & 16 & 23,77 \\
\hline Trepadeira geofítica & 2 & 1,08 & 6 & 7,17 & 9 & 8,33 \\
\hline Caméfita herbácea pulvinada & 1 & 1,11 & 1 & 1,97 & 1 & 4,34 \\
\hline Caméfita suculenta & 1 & 0,79 & 1 & 0,8 & 1 & 4,08 \\
\hline Hemicriptófita escaposa rosetada & 1 & 0,48 & 1 & 0,37 & 3 & 3,18 \\
\hline Fanerófita suculenta & - & - & 1 & 2,12 & 1 & 3,01 \\
\hline Geófita radicigema & 1 & 1,93 & 2 & 3,09 & 4 & 2,76 \\
\hline Liana fanerófita & - & - & - & - & 3 & 1,47 \\
\hline Caméfita herbácea reptante & - & - & 3 & 0,61 & 3 & 1,36 \\
\hline Epífita & 1 & 0,24 & 1 & 0,13 & 1 & 1,02 \\
\hline Total & 29 & & 41 & & 64 & \\
\hline
\end{tabular}

* $\Sigma$ de todas as formas de vida $=200 \%$.

grande importância nesta análise é o aumento em número de espécies e cobertura total ao longo da cronoseqüiência de regeneração.

Indivíduos de $A$. arenaria com ramificações a partir de órgãos subterrâneos cooperam para a formação de "ilhas de vegetação" da planta, contribuindo para o aspecto adensado que a formação de Palmae apresenta na Restinga da Marambaia. Estudos sobre germinação de sementes e influência do fogo na indução de gemas subterrâneas desta espécie contribuirão para maior compreensão das distinções fisionômicas entre as formações de Palmae no Sudeste Brasileiro e para obter-se maior conhecimento objetivando a conservação dos ambientes de restinga.

\section{Agradecimentos}

Ao comando do Campo de Provas da Marambaia (Exército Brasileiro) e ao Departamento de Botânica da UFRRJ, pelo apoio na realização deste estudo; ao 
Dr. Fábio Rubio Scarano, pelas considerações feitas durante a leitura do manuscrito; ao biólogo Marcelo da Costa Souza, pelo inestimável auxílio nas atividades de campo.

\section{Bibliografia}

Almeida, A.L. \& Araujo, D.S.D. 1997. Comunidades vegetais do cordão arenoso externo da Reserva Ecológica Estadual de Jacarepiá, Saquarema, RJ. Oecologia Brasiliensis 3: 47-63.

Alpert, P. \& Mooney, H.A. 1996. Resource heterogeneity by shrubs and topography on coastal sand dunes. Vegetatio 122: 83-93.

Andrade, M.A.B. 1971. Balanço de íons em plantas e no solo de praias arenosas litorâneas. Ciência \& Cultura 23(1): 56-56.

Araujo, D.S.D. 1992. Vegetation types of sandy coastal plains of tropical Brazil: a first approximation. Pp. 337-347. In: U. Seeliger (ed.). Coastal Plant Communities of Latin America. New York, Academic Press.

Araujo, D.S.D. \& Henriques, R.P.B. 1984. Análise florística das restingas do Estado do Rio de Janeiro. Pp. 159-193. In: L.D. Lacerda; D.S.D. Araújo; R. Cerqueira \& B. Turcq. (orgs.). Restingas: origem, estrutura, processos. Niterói, CEUFF.

Araujo, D.S.D. \& Peixoto, A.L. 1977. Renovação de uma comunidade vegetal de restinga após queimada. Pp. 1-17. In: XXVII Congresso Nacional de Botânica. Anais da Academia Brasileira de Ciências. Rio de Janeiro.

Araujo, D.S.D.; Oliveira, R.R.; Lima, E. \& Ravelli-Neto, A.1997. Estrutura da vegetação e condições edáficas numa clareira de mata de restinga na Reserva Biológica Estadual da Praia do Sul (RJ). Revista Brasileira de Ecologia 1(2): 36-43.

Bond, W.J. \& van Wilgen, B.W. 1996. Fire and plants. London, Chapman and Hall.

Brower, J.E. \& Zar, J.H. 1984. Field and laboratory methods for general ecology. $2^{\text {nd }}$ ed. Wm. Brown Company Publishers, Dubuque.

Callaway, R.M. 1995. Positive interactions among plants. Botanical Review 61: 306-349.

Carvalho, D.A. \& Oliveira-Filho, A.T. 1993. Avaliação da recomposição da cobertura vegetal de dunas de rejeito de mineração, em Mataracá/PB. Acta Botanica Brasilica 7(2): 107-117.

Castellani, T.T. \& Stubblebine, W.H. 1993. Sucessão secundária inicial em mata tropical mesófila, após perturbação por fogo. Revista Brasileira de Botânica 16(2): 181-203.

Carvalhaes, M.A. \& Mantovani, W. 1998. Florística de mata sobre restinga na Juréia, Iguape, SP. Pp. 37-48. In: Anais do IV Simpósio de Ecossistemas Brasileiros. Águas de Lindóia, ACIESP, v.2. São Paulo.

Cirne, P. \& Scarano, F.R. 1996. Rebrotamento após fogo de Andira legalis (Leguminosa) em restinga fluminense. Pp. 128-136. In: H.S. Miranda; C.H. Saito \& B.F.S. Dias (orgs.). Impactos de queimadas em áreas de cerrado e restinga. Universidade de Brasília, Brasília.
Cirne, P. \& Scarano, F.R. 2001. Resprouting and growth dynamics after fire of the clonal shrub Andira legalis (Leguminosae) in a sandy coastal plain in south-eastern Brazil. Journal of Ecology 89: 351-357.

Cirne, P.; Zaluar, H.L.T. \& Scarano, F.R. 2003. Plant diversity, interespecific association, and postfire resprouting on a sandy spit in a brazilian coastal plain. Ecotropica 9: 33-38.

Cook, R.E. 1983. Clonal plant populations. American Science 71: 244-253.

Costa e Silva, L.V. 1993. Comparação fitossociológica entre duas amostragens numa área de clareira em anos consecutivos, Estação Biológica de Caratunga, MG. Acta Botanica Brasilica 7(2): 119-127.

Coutinho, L.M. 1982. Ecological effects of fire in Brazilian cerrado. Pp. 273-291. In: B.J. Huntley \& B.H. Walker (eds.). Ecology of tropical savanas. Springer-Verlag, Berlin.

Dansereau, P. 1947. Zonation et sucession sur la restinga de Rio de janeiro. I. Halosére. Revue Canadienne de Biologie 6(3): 448-477.

De Rouw, A. 1993. Regeneration by sprouting in slash and burn rice cultivation, Taï rain forest, Côte d'Ivoire. Journal Tropical of Ecology 9: 387-408.

Denslow, J.E.D. 1980. Patterns of plant species diversity during succession under different disturbance regimes. Oecologia 46: 18-21.

Etherington, J.R. 1967. Studies of nutrient cycling and productivity in oligotrophic ecosystems. I Soil potasium and wind blown sea spray in a south in a south Wales dune grassiand. Journal of Ecology 55: 743-752.

Forman, R.T.T. \& Boerner, R.E. 1981. Fire frequency and the pine barrens of New Jersey. Bulletin of the Torrey Botanical Club 108(1): 34-50.

Frost, P.G.H.; Medina, E.; Menaut, J.C.; Solbrig, O.T.; Swift, M. \& Walker, B.H. 1986. Responses of savanas to stress and disturbance. A proposal for a Collaborative programme of research. Report of a meeting of na IUBS Working Group. Special issue 10, Biology International.

Frost, P.G.H. \& Robertson, F. 1985. The ecological effects of fire in savannas. Pp. 93-140. In: B.H. Walker (ed.). Determinants of tropical savannas. IUBS Monograph Series N. 3 ICSU Press.

Gonçalves, D.B. \& Sá, C.F.C. 1998. Dinâmica da regeneração em floresta de restinga após perturbação por tratores. Pp. 272-279. In: Anais IV Simpósio de Ecossistemas Brasileiros. Águas de Lindóia, ACIESP. v.3. São Paulo.

Goto, Y.; Yoshitake, T.; Okano, M. \& Shimada, K. 1996. Seedling regeneration and vegetative resprouting after fires in Pinus densiflora forests. Vegetatio 122: 157-165.

Hay, J.D.; Henriques, P.B. \& Costa, S.R.A. 1982. Uma avaliação preliminar da possibilidade de usar equações de regressão para estimativas da biomassa na restinga. Revista Brasileira de Botânica 5: 33-36.

Hay, J.D. \& Lacerda, L.D. 1980. Alterações nas características do solo após a fixação de Neoregelia cruenta (R. Grah.) L. B. Smith (Bromeliaceae), em um ecossistema de restinga. Ciência \& Cultura 32(7): 863-867.

Huston, M. \& Smith, T. 1987. Plant sucession: life history and competition American Naturalist 130(2): 168-198.

Kauffman, J.B. 1991. Survival by sprouting following fire in tropical forests of the eastern Amazon. Biotropica 23: 219-224. 
Köeppen, W. 1948. Climatologia. Ed. Fundo de Cultura Econômica, México.

Lacerda, L.D.; Araújo, D.S.D \& Maciel, N.C. 1993. Dry coastal ecosystems of the tropical Brazilian coast. Pp. 477-493. In: Dry coastal ecosystems. v.2. Elsevier, Amsterdam.

Leigh Jr., E.G. 1992. Por qué hay tantos tipos de árboles tropicales ? Pp. 75-99 In: E.G. Leigh Jr.; A.S. Rand, \& D.M. Windsor (eds.). Ecologia de un Bosque Tropical, ciclos estacionales y cambios a largo plazo. Colômbia, Smithsonian Institution.

Maun, M.A. 1994. Adaptations enhancing survival and establishment of seedlings on coastal dune systems. Vegetatio 111: 59-70.

Magnanini, A. 1954. Contribuição ao estudo das zonas de vegetação da praia de Sernambetiba, DF. Arquivos do Serviço Florestal 8: 147-232.

Menezes, L.F.T. \& Araujo, D.S.D. 1999. Estrutura de duas formações vegetais do cordão externo da restinga de Marambaia-RJ. Acta Botanica Brasilica 13(2): 223-235.

Menezes, L.F.T. \& Araujo, D.S.D. 2000. Variação da biomassa aérea de Allagoptera arenaria (Gomes) O. Kuntze numa comunidade arbustiva de Palmae na Restinga da Marambaia, RJ. Revista Brasileira de Biologia 60(1): 47-157.

Menezes, L.F.T.; Araujo, D.S.D. \& Goes, M.H.B. 1998. Marambaia: a última restinga carioca preservada. Ciência Hoje 136(23): 28-37.

Miranda, R.U.; Barroso, D.G.; Marinho, C.S. \& Carvalho, D.A. 1997. Estudo sobre a vegetação em dunas de rejeito de mineração no litoral norte do Estado da Paraíba. Revista Árvore 21(3): 345-351.

Moraes R.M. 1996. Allagoptera (Palmae) Pp. 1-34. In: R.M. Moraes. Flora Neotropica. New York.

Moreira, A.G. 1996. Proteção contra fogo e seu efeito na distribuição e composição de espécies de cinco fisionomias de cerrado. Pp. 112-121. In: H.S. Miranda, C.H. Saito \& B.F.S. Dias (orgs.). Impactos de queimadas em áreas de cerrado e restinga. Universidade de Brasília, Brasília.

Müeller-Dumbois, D. \& Ellenberg, H. 1974. Aims and methods of vegetation ecology. New York, John Wiley \& Sons.

Nascimento, M.T. 1996. Rapid resproutig following fire in juveniles of Metrodorea pubescens (Rutaceae) in a mesophytic forest in central Brazil. Ciência \& Cultura 48: 182-183.

Pereira, O.J. 1990. Caracterização fitofisionomica da restinga de Setiba, Guarapari, ES. Pp. 207-219 In: Anais do II Simpósio de Ecossistemas da Costa Sul e Sudeste Brasileira: estrutura, função e manejo. Águas de Lindóia, ACIESP, v.3. São Paulo.

Pereira, O.J.; Thomaz, L.D. \& Araujo, D.S.D. 1992. Fitossociologia da vegetação de antedunas da restinga de Setiba/Guarapari e em Interlagos/Vila Velha, ES. Boletim do Museu de Biologia Mello Leitão (N. Ser.) 1: 65-75.
Pornon, A.; Escaravage, N.; Till-Bottraud, I. \& Doche, B. 1997. Variations of reproductive traits in Rhododendron ferrugineum L. (Ericaceae) populations along a successional gradient. Plant Ecology 130: 1-11.

Pugnaire, S.I.; Haase, P.; Puigdefábegras, J.; Cueto, M.; Clark, S.C. \& Incoll, L.D. 1996. Facilitation and seccession under the canopy of a leguminous shrub, Retama spherocarpa, in a semi-arid environment in South-East Spain. Oikos 76: 455-464.

Ramos, A.E. \& Rosa, C.M.M. 1992. Impacto das queimadas. Pp. 34-38. In: Alternativas de desenvolvimento dos cerrados: manejo e conservação dos recursos naturais renováveis. Brasília, Fund. Pró-natura.

Rizzini, C.T. 1979. Tratado de fitogeografia do Brasil: aspectos sociológicos e florísticos. v.II. São Paulo, Hucitec-Edusp.

Sá, C.F.C. 1996. Regeneração em área de floresta de restinga na Reserva Ecológica Estadual de Jacarepiá, Saquarema/ RJ: I - Estrato herbáceo. Arquivos do Jardim Botânico do Rio de Janeiro 34(1): 177-192.

Sá, C.F.C. 2002. Regeneração de um trecho de floresta de restinga na Reserva Ecológica Estadual de Jacarepiá, Saquarema, Estado do Rio de Janeiro: II - Estrato arbustivo. Rodriguesia 53(82): 5-23.

Scarano, F.R. 2002. Structure, function and floristic relationships of plant communities in stressful habitats marginal to the brazilian atlantic rainforest. Annals of Botany 90: 517-524.

Scarano, F.R.; Cirne, P.; Trindade, Nascimento, M.T.; Sampaio, M.C.; Villela, D.M.; Wendt, T. \& Zaluar, H.L.T. 2004. Ecologia vegetal: integrando ecossistemas, comunidades, populações e organismos. Pp. 77-97. In: C.F.D. Rocha; F.A. Esteves \& F.R. Scarano. Pesquisas de longa duração na restinga de Jurubatiba. Ecologia, história natural e conservação. Rima editora. São Carlos.

Stuefer, J.F. 1994. High benefits of clonal integration in two stoloniferous species, in response to heterogeneous ligth environments. Journal of Ecology 82: 511-518.

Tabarelli, M. \& Mantovani, W. 1999. A regeneração de uma floresta tropical Montana após corte e queima (São Paulo - Brasil). Revista Brasileira de Biologia 59(2): 239-250.

Tester, J.R. 1996. Effects of fire frequency on plant species in oak savanna in east-central Minnesota. Bulletin of the Torrey Botanical Club 123(4): 304-308.

Tonlinson, P.B. 1960. Essays on the morphology of palms. II. The early growth of the palm. Principes 4(4): 140-143.

Ule, E. 1967. A vegetação de Cabo Frio. Boletim Geográfico 200: 21-32.

Whelan, R.J. 1995. The Ecology of fire. Cambridge, Cambridge University Press.

Woods, P. 1989. Effects of logging, drought, and fire on structure and composition of tropical forest in Sabah, Malaysia. Biotropica 21(4): 290-198.

Zaluar, H.L.T. \& Scarano, F.R. 2001. Facilitação em restingas de moitas: Um século de buscas por espécies focais. Pp. 3-23. In: F.A. Esteves \& L.D. Lacerda (eds.). Ecologia de restingas e lagos costeiros. Macaé, NUPEM/UFRJ. 